\title{
The Next-Generation Ground-Based Planetary Radar
}

T. Joseph W. Lazio

Jet Propulsion Laboratory, California Institute of Technology

818-354-4198

Joseph.Lazio@jpl.caltech.edu

\author{
Anne K. Virkki \\ Arecibo Observatory
}

787-878-2612 x337

anne.virkki@ucf.edu
Noemi Pinilla-Alonso

Florida Space Institute

Univ. Central Florida

npinilla@ucf.edu

L. A. M. Benner ${ }^{1}$, M. Brozovic ${ }^{1}$, B. J. Butler ${ }^{2}$, B. A. Campbell ${ }^{3}$, F. Cordova ${ }^{4}$, K. de Kleer ${ }^{5}$, P. G. Edwards ${ }^{6}$, T. M. Eubanks ${ }^{7}$, Y. R. Fernandez ${ }^{8}$, J. D. Giorgini ${ }^{1}$, E. Kruzins ${ }^{9}$, J.L. Margot ${ }^{10}$, A. Mainzer ${ }^{11}$, S. E. Marshall ${ }^{4}$, F. Paganelli ${ }^{12}$, C. A. Raymond ${ }^{1}$, P. A. Taylor ${ }^{13}$, E. Rivera-Valentín ${ }^{13}$, M. A. Slade ${ }^{1}$, F. C. F. Venditti ${ }^{4}$

${ }^{1}$ Jet Propulsion Laboratory, California Institute of Technology; ${ }^{2}$ National Radio Astronomy Observatory; ${ }^{3}$ Smithsonian Institution; ${ }^{4}$ Arecibo Observatory; Univ. Central Florida; ${ }^{5}$ California Institute of Technology; ${ }^{6}$ CSIRO Astronomy \& Space Science; ${ }^{7}$ Space Initiatives Inc.; ${ }^{8}$ Univ. Central Florida; ${ }^{9}$ Canberra Deep Space Communications Center; ${ }^{10}$ Univ. California, Los Angeles; ${ }^{11}$ Univ. Arizona; ${ }^{12}$ Green Bank Observatory; ${ }^{13}$ Lunar \& Planetary Institute, Universities Space Research Association
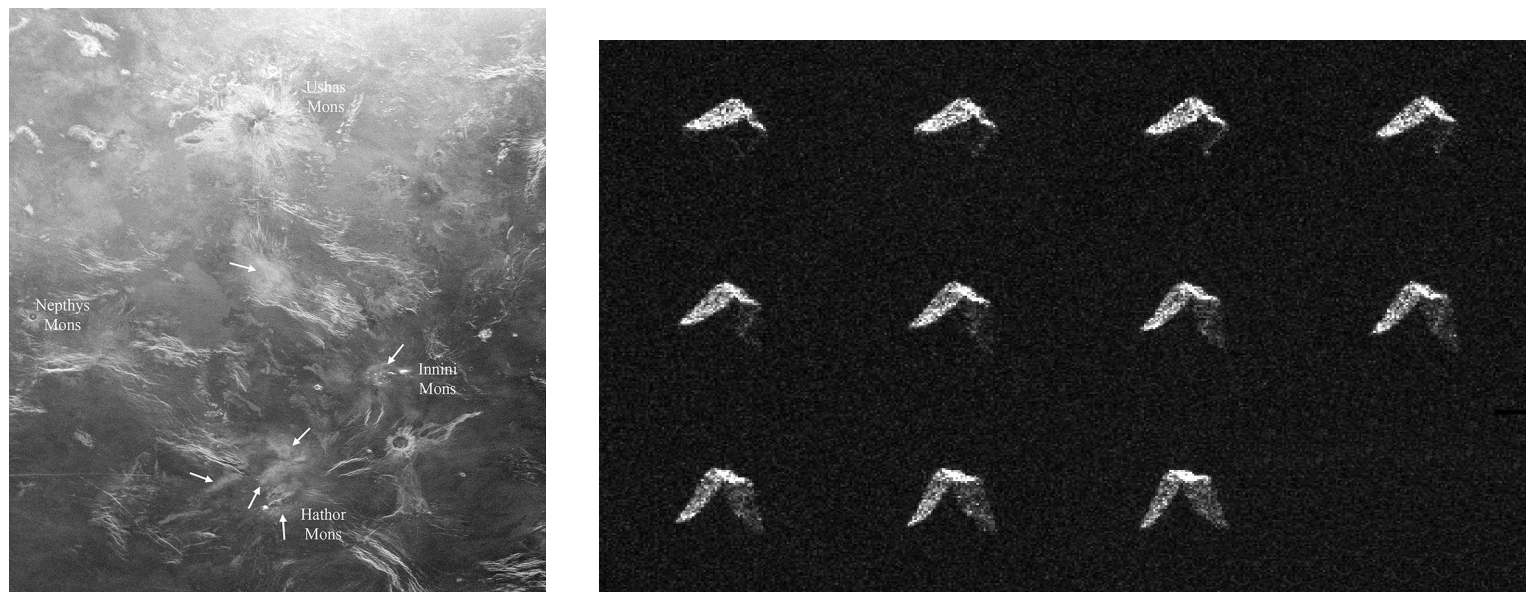

(Left) Arecibo radar image of Dione Regio on Venus. White arrows indicate radar-bright features that potentially represent debris from previous volcanic eruptions (Campbell et al. 2017). (Right) Goldstone Solar System Radar image of the near-Earth asteroid (NEA) $2017 B Q_{6}$ showing sharp facets on this object. NEAs display a range of surface features, from which evolution and collisional processes can be constrained, and are important to characterize for planetary defense.

Part of this research was carried out at the Jet Propulsion Laboratory, California Institute of Technology, under a contract with the National Aeronautics and Space Administration. The Arecibo Planetary Radar Program is supported by the National Aeronautics and Space Administration's Near-Earth Object Observations Program through Grant No. 80NSSC19K0523 awarded to the University of Central Florida. The Arecibo Observatory is a facility of the National Science Foundation operated under cooperative agreement by University of Central Florida, Yang Enterprises, Inc., and Universidad Ana G. Méndez. This whitepaper reflects ideas and advice from the participants in the Next-Generation Planetary Radar workshop organized by the W. M. Keck Institute for Space Studies. Some of the information presented in this white paper is pre-decisional and is for discussion and planning purposes only. 


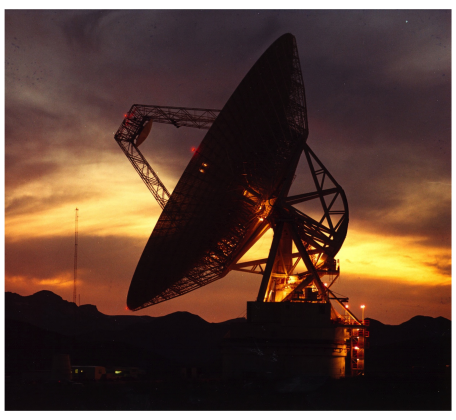

Goldstone Solar System Radar

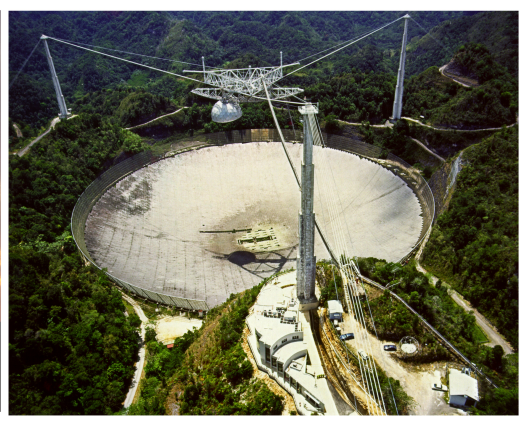

Arecibo Planetary Radar

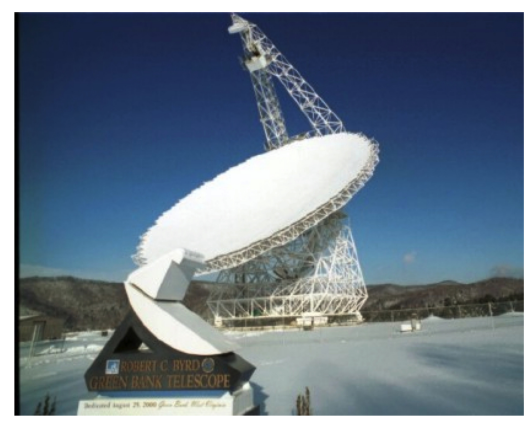

Green Bank Telescope (receive only)

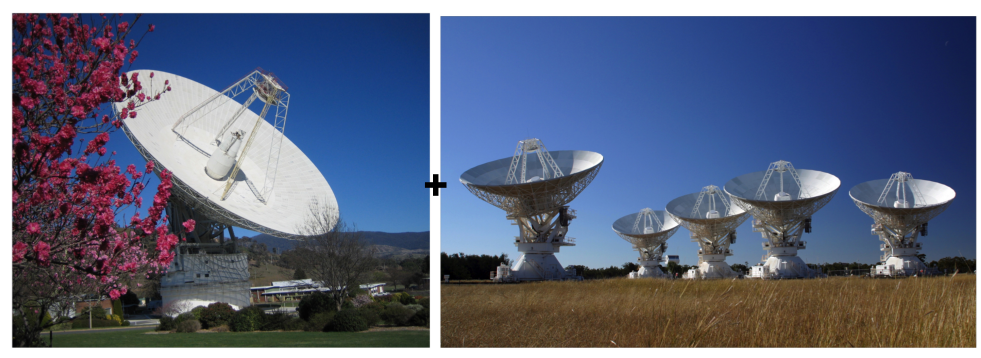

Canberra + Australia Telescope Compact Array (bistatic)

Figure 1: The international planetary radar infrastructure has enabled a variety of discoveries about all terrestrial planets, the largest moons, and near-Earth asteroids.

This white paper complements previously-submitted white papers focusing on near-Earth asteroids, small bodies, large Solar System bodies, and planetary defense, most notably those by Binzel et al., Davidsson et al., Mainzer et al., Margot, Milam et al., Paganelli et al., Rivera-Valentín et al., Stickle et al., Taylor et al., and Virkki et al. It also reflects broader discussions during the "Next-Generation Ground-Based Planetary Radar" workshop at the W. M. Keck Institute for Space Studies (KISS). ${ }^{1}$

This white paper contains two key points: (1) In the near term, maintenance of the current planetary radar infrastructure is required. (2) Over the next decade, a variety of technologies - arrays of transmitting antennas; modular, solid-state microwave amplifiers; and improved data acquisition, processing, data fusion, and archiving - will mature and could be deployed to produce a much more capable and reliable ground-based radar for planetary science and planetary defense.

\section{Science Goals}

This listing is based substantially on community discussions at the KISS workshop and is intended to be illustrative and deliberately ambitious, but is not exhaustive.

Near-Earth Asteroids (NEAs) Expanding ground-based planetary radar capabilities to be able to discover NEAs to 10 lunar distances would be revolutionary. Further, an expansion of capability in the coming decade also would produce many more opportunities for

\footnotetext{
${ }^{1}$ https://www.kiss.caltech.edu/programs.html\#radar
} 
ground-based radar studies of NEAs, both to characterize their properties and for planetary defense, to keep pace with an accelerating discovery rate from a combination of ground- and space-based survey programs. New observational modes, e.g., based on synthetic aperture radar techniques, could also yield new insights into surface properties.

Venus Radar observations provide crucial information about Venus' surface and interior properties, with implications for models of its formation and evolution and for the properties of extrasolar planets (Margot et al. 2019; Campbell et al. 2019a, b). Because they obtain much longer time scale coverage and can search for temporal changes, ground-based planetary radar observations are a powerful complement to missions. Of particular value would be to improve the spatial resolution from the current $\mathrm{km}$-scale capabilities to be comparable to that of orbiting missions (e.g., $\sim 150 \mathrm{~m}$, Magellan)

Outer Planet Satellites and Ocean Worlds Ground-based radar can probe deeply into ice layers, depending upon the amount of rocky debris present. Like the case of Venus, ground-based planetary radars also can provide longer time coverage than missions. Improving the capability to probe the satellites of Jupiter and Saturn at higher signal-to-noise ratios and reach the Uranian satellites would offer the possibility of monitoring for seasonal variations and data fusion with other facilities and missions.

"Mini-moons" Small NEAs have been discovered on metastable orbits (e.g., $2006 \mathrm{RH}_{120}$; $2020 \mathrm{CD}_{3}$, discovered in February). Because of their extended residence relatively close to the Earth, they present opportunities for more detailed studies than NEAs that have shortduration apparitions as well as opportunities for studies of their dynamics and as possible targets for rapid technology demonstration missions. Ground-based planetary radars could be a powerful means of studying these objects, however, the discovered objects have been small $(\lesssim 10 \mathrm{~m})$. More powerful transmitters would increase the number of observational opportunities.

Finally, while not providing direct science measurements, the planetary radar infrastructure has assisted in the recovery of spacecraft, ${ }^{2}$ thereby ensuring that missions could continue science measurements.

\section{Technical Overview}

During a planetary radar experiment, a signal with predefined characteristics is transmitted. The signal interacts with and is modified by the target object. The changes observed when the reflected signal is received are used to make inferences about the target object: its precise location and motion along with its size, shape, spin, and reflectivity, which in turn constrains its surface properties, gravity, and potentially composition. The information returned can be comparable to spacecraft missions at costs that permit sampling a larger fraction of the NEA population in order to direct spacecraft to the most scientifically interesting objects.

The existing planetary radar infrastructure (Figure 1 and Table 1) can observe all terrestrial planets, the larger Main Belt Asteroids, the largest moons of Jupiter and Saturn (e.g.,

\footnotetext{
2 "SOHO spacecraft located with ground-based radar," https ://sohowww. nascom.nasa.gov/newsroom/oldesapr/press27.html
} 
Muhleman et al. 1990; Campbell et al. 2003), and NEAs at distances of order 0.1 au, with typical NEAs being several hundred meters in diameter. Smaller NEAs can be observed at smaller distances, with objects of order $10 \mathrm{~m}$ and smaller having been observed at distances less than 1 lunar distance. With its high gain and large transmit power, Arecibo can detect targets at the largest distances. However, Arecibo has a limited declination range and can observe only approximately $30 \%$ of the sky (centered approximately on a declination of $18^{\circ}$ ). By contrast, the GSSR, either monostatically or bistatically with the GBT, can observe approximately $75 \%$ of the sky. Naidu et al. (2016) provide further technical details and comparisons between the various facilities, but, notably, most of this observational capability is based in the United States.

Table 1: Existing Planetary Radar Infrastructure

\begin{tabular}{|c|c|c|c|c|}
\hline System & Antenna & $\begin{array}{l}\text { Transmitter } \\
\text { Power }\end{array}$ & $\begin{array}{c}\text { Transmit } \\
\text { Frequency / } \\
\text { Wavelength }\end{array}$ & Gain \\
\hline $\begin{array}{l}\text { Arecibo Planetary } \\
\text { Radar }\end{array}$ & $305 \mathrm{~m}$ diameter & $900 \mathrm{~kW}$ & $\begin{array}{c}2.38 \mathrm{GHz} \\
12.6 \mathrm{~cm}\end{array}$ & $73.0 \mathrm{dBi}$ \\
\hline $\begin{array}{l}\text { Goldstone Solar } \\
\text { System Radar }\end{array}$ & $70 \mathrm{~m}$ diameter & $450 \mathrm{~kW}$ & $\begin{array}{l}8.56 \mathrm{GHz} \\
3.5 \mathrm{~cm}\end{array}$ & $73.7 \mathrm{dBi}$ \\
\hline Green Bank Telescope & $100 \mathrm{~m}$ diameter & $\begin{array}{c}\sim 100 \mathrm{~kW} \\
\text { proposed }\end{array}$ & $\begin{array}{l}35 \mathrm{GHz} \\
1 \mathrm{~cm}\end{array}$ & $89.7 \mathrm{dBi}$ \\
\hline $\begin{array}{l}\text { Southern } \\
\text { Hemisphere }\end{array}$ & $\begin{array}{l}70 \mathrm{~m} \text { diameter transmit } \\
+50 \mathrm{~m} \text { equivalent receive }\end{array}$ & $\begin{array}{l}20 \mathrm{~kW} \text { current } \\
80 \mathrm{~kW}(2021)\end{array}$ & $\begin{array}{l}7.19 \mathrm{GHz} \\
4.2 \mathrm{~cm}\end{array}$ & $72.5 \mathrm{dBi}$ \\
\hline $\begin{array}{l}\text { Deep Space Station-13 } \\
\text { (Goldstone) }\end{array}$ & $34 \mathrm{~m}$ diameter & $80 \mathrm{~kW}$ & $\begin{array}{l}7.19 \mathrm{GHz} \\
4.2 \mathrm{~cm}\end{array}$ & $67.0 \mathrm{dBi}$ \\
\hline
\end{tabular}

In a development from last decade, there is an initial Southern Hemisphere capability, formed by a bistatic combination of the $70 \mathrm{~m}$ Deep Space Station-43 (DSS-43) antenna at the DSN's Canberra Complex and the Australia Telescope Compact Array (ATCA). The Southern Hemisphere system enables full-sky coverage, which is particularly valuable for tracking closely-approaching or impacting NEAs, ${ }^{3}$ albeit DSS-43 has a lower power transmitter than DSS-14/GSSR. Notably, an imminent impactor would not have significant sky motion on its final dive toward Earth, necessitating global coverage for enhanced warning.

In the next decade, the intermediate frequency component of the Square Kilometre Array Phase 1 (SKA1-Mid), to be sited in South Africa, is planned to be capable of receiving at the transmit frequencies of the DSN's Canberra Complex (Jones \& Lazio 2015), but there will be only limited common sky visibility. In the northern hemisphere, the proposed next-generation Very Large Array (ngVLA), when operational in the 2030s, would offer frequency coverage that overlaps with both the Arecibo Planetary Radar and the GSSR transmitters and considerable mutual visibility (Brozovic et al. 2018).

\section{Organization}

Arecibo Observatory A facility of the NSF, for which the current cooperative agreement provides for a decreasing amount of funding. NASA provides funding for the operation and

\footnotetext{
${ }^{3}$ At its closest approach, about 40000 km, 2012 TC $_{4}$ was over Australia.
} 
maintenance of the Arecibo Planetary Radar.

Deep Space Network Operates and maintains both the GSSR and the Canberra Complex, with funding from NASA.

Green Bank Telescope A facility of the NSF, for which the current cooperative agreement provides for a decreasing amount of funding. In order to ensure continued availability of the GBT for radar studies, funds would need to be allocated for dedicated GBT time.

There also have been "radar speckle" observations of asteroids (Busch et al. 2010), which provide information on body spin-states, conducted with the Very Large Array (VLA) and the Very Long Baseline Array (VLBA), both operated by the National Radio Astronomy Observatory (NRAO) on behalf of the NSF.

For southern hemisphere observations, the Australia Telescope National Facility (ATNF) operates and maintains the ATCA, with funding support from the Australian Commonwealth Scientific \& Industrial Research Organisation (CSIRO). The recent establishment of an Australian Space Agency may represent additional opportunities for support of a southern hemisphere radar system.

\section{Technology Development and Paths Forward}

Observing NEAs at larger distances, reaching deeper into the outer Solar System, and other ambitious science goals of $\S 1$ and the KISS workshop can be achieved with a combination of technologies to be matured in the coming decade. Traditional single-aperture ground-based planetary radars obtain a signal-to-noise $(\mathrm{S} / \mathrm{N})$ given by the radar equation,

$$
\mathrm{S} / \mathrm{N} \propto \frac{G_{\mathrm{RX}} P_{\mathrm{TX}} G_{\mathrm{TX}}}{R^{4}}
$$

where $G_{\mathrm{RX}}$ and $G_{\mathrm{TX}}$ are the gains of the receiving and transmitting antennas, respectively; $P_{\text {TX }}$ is the power of the transmitter; and $R$ is the distance to the target. For an antenna of diameter $D, G \propto D^{2}$.

Gain and Antenna Arrays A significant improvement in $G_{\mathrm{RX}}$ or the combination $P_{\mathrm{TX}} G_{\mathrm{TX}}$ could be obtained via antenna arrays. Current single dish antennas are at the scale that larger antennas are not a viable approach to obtain significant improvements in antenna gain: No fully-steerable single dish antenna significantly larger than the GBT has since been constructed since its completion in the early 2000s. The continued operation of the existing large single dish antennas is therefore critical for a near-term viable planetary radar capability.

For a transmitting array of $N$ identical antennas (each with $P_{1} G_{1}$ ), the net transmit capability is $N^{2} P_{1} G_{1}$. Thus, an array of $N$ smaller antennas with lower power transmitters can equal or exceed the capabilities of the current large-diameter antennas. As a specific example, $200 \times 18 \mathrm{~m}$ fully-steerable antennas each equipped with a $20 \mathrm{~kW}$ transmitter is approximately $5 \times$ Arecibo; for reference, the ngVLA plans 244 antennas, each of $18 \mathrm{~m}$ diameter. Phased arrays are, of course, standard for terrestrial radar applications, and there 
have been limited DSN experiments of phasing transmit antennas (the DSN "Uplink Array," e.g., Vilnrotter et al. 2006; D'Addario et al. 2009). These experiments demonstrated the expected $N^{2}$ improvements for coherent addition of antennas, but they were not required to demonstrate the range (distance) and range-rate (Doppler) precision required for ground-based planetary radar. Additional work is necessary to verify that the required precisions can be obtained.

Interferometric ("receive-only") arrays are standard for radio astronomical telescopes and larger receiving gains $G_{\mathrm{Rx}}$ could obtained via bistatic operations with future radio astronomical arrays, such as the SKA1-Mid or the ngVLA.

Transmitter Power and Reliability More reliable microwave amplifiers could be modular systems, consisting of many, lower power solid-state amplifiers, the outputs of which would be combined coherently. Fundamental to both the Arecibo Planetary Radar and the GSSR are klystrons - high-power, vacuum tube-based microwave amplifiers. Planetary radar klystrons have power levels and power densities that are orders of magnitude higher than all other applications (e.g., military radars, some medical devices, particle accelerators). As a consequence, current planetary radar klystrons are at engineering limits of reliability, which have affected the availability of both Arecibo and the GSSR over the past decade, and the time to assemble one can be comparable to its lifetime $(\sim 1 \mathrm{yr})$.

Modular solid-state systems achieving $1 \mathrm{~kW}$ to $20 \mathrm{~kW}$ are being demonstrated, consisting of several to tens of lower power solid-state amplifiers being combined coherently, and they offer the clear benefit of graceful degradation. Yet to be demonstrated is that these systems can be scaled to $100 \mathrm{~kW}$ or more, while maintaining a high efficiency of coherent combination of the signals.

Data Acquisition, Processing, Data Fusion, and Archiving Multiple avenues exist for improving all stages of ground-based planetary radar observations. Existing tools already scan Minor Planet Center (MPC) reports on an hourly cadence to assess the detectability of newly-discovered NEAs by one of the ground-based planetary radar facilities. If automatic scheduling and remote observation tools were developed, automated and rapid NEA radar observations could be obtained.

Shape models of asteroids are a key outcome of ground-based planetary radar observations and are of value to both science investigations and planetary defense. Constructing such shape models currently requires substantial human oversight, due to degeneracies in the delay-Doppler radar data, leading to substantial backlogs of data. The NASA Frontier Development Lab has conducted proof-of-concept investigations into machine learning approaches for asteroid shape modeling, including using Bayesian optimization to find pole direction with less human oversight and using neural networks to rapidly provide initial approximations of the shape.

Data fusion from observations at different wavelengths could yield new insights on surface and near-surface properties of small bodies, notably including the Galilean satellites. There have been combinations of radar observations with visible or near-infrared wavelength images, but facilities such as the Atacama Large Millimeter/submillimeter Array (ALMA) and missions such as the Europa Clipper and the JUpiter ICy moons Explorer (JUICE) 
expand the wavelength coverage to be considered and the characteristics of the material to be constrained.

High-level data products from ground-based planetary radar observations are provided to the Planetary Data System (PDS). Traditionally, this process has been fairly laborintensive, but recent developments both at the PDS and the ground-based planetary radar have been streamlining this archiving. Nonetheless, additional steps will be required before routine and automated archiving of ground-based planetary radar data is obtained.

\section{Paths Forward}

Over the course of the next decade, the following changes and improvements are feasible, provided that sufficient funding is maintained. In what follows, we assume that the current technology developments in the areas of solid-state amplifiers and antenna arrays mature in the first half of the decade.

- The Arecibo telescope has suffered recent damage, but funding is available for repairs and to restore functionality. The GSSR has been offline due to repair and upgrade of its klystrons; but new klystrons are arriving, bistatic GSSR-GBT tracks on Venus were performed in early September, and its return to a $450 \mathrm{~kW}$ transmit capability is expected in early 2021.

- The DSN is upgrading the DSS-43 transmitter (at the Canberra Complex) from its current $20 \mathrm{~kW}$ capability to $80 \mathrm{~kW}$, with a return to service in 2021. Further, all current demonstrations have been continuous wave (CW) transmissions only, allowing rotation periods and surface characteristics of NEAs to be determined, but no orbit determination. Part of the on-going DSS-43 upgrade will enable the injection of arbitrary radar waveforms, enabling ranging modulations and delay-Doppler imaging capabilities. Second, a design exists for an Ultra-Wideband-Mid receiving system at the Parkes Radio Telescope. This receiving system would include coverage of the transmission frequencies of the DSN Canberra Complex antennas. The resulting increase in receiving antenna diameter, from the ATCA (50 m equivalent) to Parkes (64 m diameter) would produce a $50 \%$ increase in the receiving antenna gain $G_{\mathrm{RX}}$ (eqn. 1).

- The DSN has a notional schedule for the installation of a new, solid-state transmitter for the GSSR in 2027. If there are no changes to this current notional schedule, the GSSR transmitter would be substantially more robust. On this time scale, one option would be to replace the GSSR klystrons with solid-state amplifiers.

- If the GBT is equipped with a Ka-band transmitter (Bonsall et al. 2019) and it were to be paired with the ngVLA in the 2030s, the pair would be a powerful bistatic combination. Imaging of the outer planet satellites would be among the scientific opportunities that could be realized.

Design of an antenna array-based system could begin in the first half of the decade, provided that support is available from NASA and the NSF. An array of 18 mdiameter antennas could potentially leverage other developments in antenna manufacturing. 
As noted above, it would be outfitted with lower power systems, which would be substantially higher reliability, and it would be fully steerable. This sequence of events would lead to a next-generation planetary radar system, with the objective of increasing science capability, reliability, and providing more effective planetary defense.

\section{References}

Bonsall, A., et al. 2019, "GBT Planetary Radar System," Astro2020 Science White Paper Brozovic, M., Butler, B. J., Margot, J.-L., et al. 2018, "Planetary Bistatic Radar," in Science with a Next Generation Very Large Array, eds. E. Murphy and the ngVLA Science Advisory Council (Astronomical Society of the Pacific: San Francisco) p. 113; ISBN: 978-1-58381-919-7

Busch, M. W., Kulkarni, S. R., Brisken, W., et al. 2010, "Determining asteroid spin states using radar speckles," Icarus, 209(2), 535; doi: 10.1016/j.icarus.2010.05.002

Campbell, B. A., Campbell, D. B., Carter, L. M., et al. 2019b, "The Mean Rotation Rate of Venus from 29 Years of Earth-based Radar Observations," Icarus, 332, 19; doi: 10.1016/j.icarus.2019.06.019

Campbell, B., Neish, C., Campbell, D., et al. 2019a, "Radar Astronomy for Planetary Surface Studies," Astro2020 Science White Paper

Campbell, B. A., Morgan, G. A., Whitten, J. L., Carter, L. M., Glaze, L. S., \& Campbell, D. B. 2017, "Pyroclastic flow deposits on Venus as indicators of renewed magmatic activity," J. Geophys. Res. Planets, 122, 1580; doi: 10.1002/2017JE005299

Campbell, D. B., Black, G. J., Carter, L. M., \& Ostro, S. J. 2003, "Radar Evidence for Liquid Surfaces on Titan," Science, 302, 431; doi: 10.1126/science.1088969

D'Addario, L., Proctor, R., Trinh, J.., et al. 2009, "Uplink Array Demonstration with Ground-Based Calibration," Interplanetary Network Progress Report (Jet Propulsion Laboratory, California Institute of Technology), 42-176, 1

Jones, D. L., \& Lazio, J. 2015, "Enhancing Science from Future Space Missions and Planetary Radar with the SKA," in Advancing Astrophysics with the Square Kilometre Array, eds. T. Bourke et al. (SKA Organization, Dolman Scott Ltd.: Manchester, UK) p. 1911; ISBN 978-1-909204-71-3

Margot, J.-L., Campbell, D. B., Padovan, S., Slade, M. A., Ghigo, F., \& Bonsall, A. 2019, "Structure of terrestrial planets and ocean worlds," Astro2020 Science White Paper, Bull. Amer. Astron. Soc., 51, 120

Muhleman, D. O., Grossman, A. W., Butler, B. J., \& Slade, M. A. 1990, "Radar Reflectivity of Titan," Science, 248, 975; doi: 10.1126/science.248.4958.975

Naidu, S. P., Benner, L. A. M., Margot, J.-L., Busch, M. W., \& Taylor, P. A. 2016, "Capabilities of Earth-based Radar Facilities for Near-Earth Asteroid Observations," AJ, 152, 99; doi: 10.3847/0004-6256/152/4/99

Vilnrotter, V., Lee, D., Cornish, T., et al. 2006, "Uplink Arraying Experiment with the Mars Global Surveyor Spacecraft," Interplanetary Network Progress Report (Jet Propulsion Laboratory, California Institute of Technology), 42-166, 1 